\title{
Hydrothermal stability of ordered mesoporous titanosilicate materials prepared at room temperature
}

\section{${ }^{a}$ Cristina Galacho, ${ }^{b}$ Manuela. M. L. Ribeiro Carrott, ${ }^{\text {c P.J.M. Carrott and }{ }^{d} \text { I.P.P. }}$ Cansado}

\author{
Centro de Química de Évora e Departamento de Química da Universidade de Évora, \\ Rua Romão Ramalho $n^{\circ} 59,7000-671$ Évora, Portugal \\ apcg@uevora.pt, 'bmanrc@uevora.pt, ${ }^{\mathrm{c}}$ peter@uevora.pt, ${ }^{\mathrm{a} i p p c @ u e v o r a . p t, ~}$
}

Keywords: Room temperature synthesised Si-MCM-41 and Ti-MCM-41, hydrothermal stability, XRD, Nitrogen adsorption; DR UV-Vis.

\begin{abstract}
A study of hydrothermal stability, performed in boiling water under static conditions, of MCM-41 materials containing different titanium content, prepared by direct synthesis at ambient temperature and pressure, using tetraethoxysilane, titanium ethoxide and octadecyltrimethylammonium bromide is presented. The behaviour is compared with pure silica grades prepared by a similar procedure. The samples were characterised by X-ray diffraction, adsorption of nitrogen at $77 \mathrm{~K}$ and diffuse reflectance UV-Vis spectroscopy. It was found that the stability improves as the amount of titanium increases and that $\mathrm{Ti}-\mathrm{MCM}-41$ samples prepared with $\mathrm{Si} / \mathrm{Ti} \leq 50$ are significantly stable. After $12 \mathrm{~h}$ in boiling water the pore size uniformity was practically maintained and only a small decrease in pore volume (5-9\%), total surface area $(2-7 \%)$ and mesopore width $(3 \%)$ and a slight increase in pore wall thickness (3-6\%) occurred. In contrast, some degradation of the MCM41 structure for the pure silica and the lower $\mathrm{Ti}$ content $(\mathrm{Si} / \mathrm{Ti}=100)$ samples was observed with the effect being less pronounced for the latter. The higher hydrothermal stability of titanium substituted samples is probably correlated with a higher degree of polymerisation of the pore walls and with the presence of extra framework titanium.
\end{abstract}

\section{Introduction}

Since the first publications on the M41S family of silicate and aluminosilicate mesoporous molecular sieves, disclosed by the scientists of the Mobil Corporation Strategic Research Center [1,2] ordered mesoporous materials have stimulated great interest in the scientific community, principally, in the fields of catalysis and materials science [3-9]. The Mobil Composition of Matter No. 41, MCM-41 material, with an hexagonal array of unidirectional tubular pores and other important features such as extremely high surface area and porosity, narrow pore size distributions and pore size adjustable from $\sim 2$ to $10 \mathrm{~nm}$ is one of the ordered mesoporous structures most studied. It is well known that pure silicas are catalytically inactive. However, catalytic activity, acid or redox, can be generated by modification of the siliceous framework by heteroelements such as $\mathrm{Al}$ and Ti. The synthesis of Ti-MCM-41 was reported for the first time in $1994[10,11]$ and it became extremely important, from the point of selective oxidation reactions, because it opened new possibilities for conversion of bulky substrates. Nevertheless, this and other fields of application can be substantially reduced due to lack of stability under process conditions. Therefore, the structural stability namely, thermal, mechanical and hydrothermal, of mesoporous titanosilicates are key factors in their practical applications.

Various authors have investigated the hydrothermal stability of purely siliceous MCM-41 [7,12-15] as well as of metal substituted MCM-41 materials [16-19]. The improvement of hydrothermal stability of those mesoporous materials has also been subject to extended research [20-24]. However, there are relatively few publications in the literature related to the hydrothermal stability of Ti-MCM-41 materials. Rhee et al. [25] reported that the Ti-MCM-41 structure collapsed under mild reaction conditions for phenol hydroxylation with $\mathrm{H}_{2} \mathrm{O}_{2}$. F.-S. Xiao et al. [18] reported the collapse of the ordered hexagonal structure of Ti-MCM-41 materials after treatment in boiling 